\title{
BOUNDARY FUNCTIONS FOR BOUNDED HARMONIC FUNCTIONS
}

\author{
BY \\ T. J. KACZYNSKI
}

Let $D$ be the open unit disk in the complex plane and let $C$ be its boundary, the unit circle. If $\zeta \in C$, then by an arc at $\zeta$ we mean a simple arc $\gamma$ with one endpoint at $\zeta$ such that $\gamma-\{\zeta\} \subseteq D$. In this paper we use the term bounda $-y$ function in the following sense. If $f$ is a function defined in $D$ and $\phi$ is a function defined on a set $S \subseteq C$, then we say that $\phi$ is a boundary function for $f$ if, and only if, for each $\zeta \in S$ there exists an $\operatorname{arc} \gamma$ at $\zeta$ such that $f(z)$ approaches $\phi(\zeta)$ as $z$ approaches $\zeta$ along $\gamma$. It is known that if $\phi$ is a boundary function for a continuous function, then $\phi$ can be made into a function of Baire class 0 or of Baire class 1 by changing its values on at most a countable set of points [4, Theorems 2, 3], [6, Theorem 6], [8, Theorem 3]. Hence $\phi$ is of Baire class at most 2. Conversely, if $\phi$ is a function defined on $C$ such that $\phi$ can be made into a function of Baire class 0 or 1 by changing its values on at most a countable set, then $\phi$ is a boundary function for some continuous function [1, Theorem 8]. Bagemihl and Piranian gave an example [1, Theorem 6] of a harmonic function having a boundary function defined on $C$ that is not of Baire class 0 or 1 , and they asked [1, Problem 5] whether there exists a bounded harmonic function having a boundary function defined on $C$ that is not of Baire class 0 or 1 . In the present paper we answer this question by constructing the desired function. We then show that, despite this example, a boundary function for a bounded harmonic function always resembles a function of Baire class 0 or 1 in this respect: its set of discontinuity points is of the first category.

We say that a function $f$ defined in $D$ has the asymptotic value $a$ at a point $\zeta \in C$ if there exists an $\operatorname{arc} \gamma$ at $\zeta$ such that $f(z)$ approaches $a$ as $z$ approaches $\zeta$ along $\gamma$. We say that $f$ has general limit $a$ at $\zeta$ if $f(z)$ approaches $a$ as $z$ approaches $\zeta$ with no restrictions other than that $z \in D$.

Let $P_{r}(\theta)$ denote the Poisson kernel; that is,

$$
P_{r}(\theta)=\frac{1-r^{2}}{1+r^{2}-2 r \cos \theta}
$$

We list three facts that we will use about the Poisson integral. These facts are presumably well known, and in any case can be easily derived from the discussion on pp. 32-38 of [3].

Received by the editors October 16, 1967 and, in revised form, January 2, 1968. 
(i) If $\phi$ is a bounded measurable real-valued function on $C$, then the Poisson integral of $\phi$, defined in $D$ by

$$
f\left(r e^{i \theta}\right)=\frac{1}{2 \pi} \int_{-\pi}^{\pi} \phi\left(e^{i t}\right) P_{r}(\theta-t) d t
$$

is a bounded harmonic function in $D$.

(ii) If $\phi$ is a measurable function defined almost everywhere on $C$, if

$$
\int_{-\pi}^{\pi}\left|\phi\left(e^{i t}\right)\right| d t<\infty
$$

and if $\phi$ is defined and continuous at a point $\zeta_{0} \in C$, then the Poisson integral of $\phi$ has general limit $\phi\left(\zeta_{0}\right)$ at $\zeta_{0}$.

(iii) If $f$ is harmonic in $D$, and if, for some $p>1$,

$$
\left\{\int_{-\pi}^{\pi}\left|f\left(r e^{i t}\right)\right|^{p} d t: r \in[0,1)\right\}
$$

is a bounded set of numbers, then the radial limit of $f$ exists at almost every point of $C$, and if $\phi$ is the function defined almost everywhere on $C$ by this radial limit, then $\int_{-\pi}^{\pi}\left|\phi\left(e^{i t}\right)\right| d t<\infty$ and $f$ is the Poisson integral of $\phi$.

Before constructing our function, we prove a simple lemma.

LEMMA. Let $\left\{I_{n}\right\}_{n=1}^{\infty}$ be a sequence of pairwise disjoint closed arcs of $C$, each arc containing more than one point. Let $\left\{\Omega_{n}\right\}_{n=1}^{\infty}$ be a sequence of Jordan domains in $D$ satisfying the following conditions.

$$
\begin{gathered}
\bar{\Omega}_{n} \cap C=I_{n} . \\
n \neq m \Rightarrow \bar{\Omega}_{n} \cap \bar{\Omega}_{m} \text { is empty. } \\
\text { diameter } \Omega_{n} \rightarrow 0 \text { as } n \rightarrow \infty .
\end{gathered}
$$

Let $I_{n}^{*}$ denote the interior (relative to $C$ ) of $I_{n}$. Then each point of $C-\bigcup_{n=1}^{\infty} I_{n}^{*}$ is accessible by a simple arc in $D-\bigcup_{n=1}^{\infty} \bar{\Omega}_{n}$.

Proof. Let $\Gamma_{n}=\left(\right.$ boundary of $\left.\Omega_{n}\right)-I_{n}^{*}$. Let

$$
\Gamma=\left(C-\bigcup_{n=1}^{\infty} I_{n}^{*}\right) \cup \bigcup_{n=1}^{\infty} \Gamma_{n}
$$

Let $h_{n}$ be a homeomorphism of $I_{n}$ onto $\Gamma_{n}$ that fixes each endpoint of $I_{n}$. Define functions $\phi$ and $\phi_{p}(p=1,2,3, \ldots)$ on $C$ by setting

$$
\begin{aligned}
\phi(\zeta)=\zeta & \text { if } \zeta \in C-\bigcup_{n=1}^{\infty} I_{n}, \\
\phi(\zeta)=h_{n}(\zeta) & \text { if } \zeta \in I_{n}, \\
\phi_{p}(\zeta)=\zeta & \text { if } \zeta \in C-\bigcup_{n=1}^{p} I_{n}, \\
\phi_{p}(\zeta)=h_{n}(\zeta) & \text { if } \zeta \in I_{n} \text { and } 1 \leqq n \leqq p
\end{aligned}
$$


Obviously $\phi_{p}$ is continuous. Moreover,

$$
\begin{array}{ll}
\left|\phi_{p}(\zeta)-\phi(\zeta)\right|=0 & \text { if } \zeta \notin \bigcup_{n=p+1}^{\infty} I_{n}, \\
\left|\phi_{p}(\zeta)-\phi(\zeta)\right| \leqq \text { diameter of } \Omega_{n} & \text { if } \zeta \in I_{n} \text { and } n \geqq p+1 .
\end{array}
$$

Since (diameter of $\Omega_{n}$ ) $\rightarrow 0$ as $n \rightarrow \infty$, it follows that $\phi_{p} \rightarrow \phi$ uniformly on $C$, so that $\phi$ is continuous. It is clear that $\phi$ is one-to-one and onto $\Gamma$, and since the inverse of a one-to-one continuous function on a compact set is always continuous, it follows that $\phi$ is a homeomorphism between $C$ and $\Gamma$. Thus $\Gamma$ is a Jordan curve. It is not hard to show that each point of $\Omega_{n}$ can be joined by an arc not meeting $\Gamma$ to some point outside of $\bar{D}$, so each $\Omega_{n}$ is contained in the unbounded domain determined by $\Gamma$. Therefore, if $B$ denotes the bounded domain determined by $\Gamma$, then $B \subseteq D-\bigcup_{n=1}^{\infty} \bar{\Omega}_{n}$. But, by the corollary on p. 164 of [7], each point of $\Gamma$ is accessible by a simple arc in $B$, so the lemma is proved.

If $S$ is a set of real numbers, let expi $S$ denote $\left\{e^{i t}: t \in S\right\}$. If $S$ and $T$ are sets of real numbers, let

$$
\Delta(S, T)=\inf \{|s-t+2 m \pi|: s \in S, t \in T, \text { and } m \text { is an integer }\} .
$$

If $y$ is a real number, let $\Delta(y, T)$ mean $\Delta(\{y\}, T)$.

Let $\left\{\rho_{n}\right\}_{n=1}^{\infty}$ be a countable dense subset of $(-\pi, \pi)$. We inductively construct a sequence of natural numbers $\{n(k)\}_{k=1}^{\infty}$ and a sequence of real numbers $\left\{\varepsilon_{k}\right\}_{k=1}^{\infty}$ in such a way that the following conditions are satisfied.

I. $0<\varepsilon_{k} \leqq 1 / 2^{k+1}$.

II. $-\pi<\rho_{n(k)}-\pi \varepsilon_{k}^{2} / 2<\rho_{n(k)}+\pi \varepsilon_{k}^{2} / 2<\pi$.

Set

Then we want

$$
\alpha(k)=\rho_{n(k)}-\pi \varepsilon_{k}^{2} / 2, \quad \beta(k)=\rho_{n(k)}+\pi \varepsilon_{k}^{2} / 2 .
$$

III. $h \neq k \Rightarrow[\alpha(h), \beta(h)] \cap[\alpha(k), \beta(k)]$ is empty.

IV. $\varepsilon_{k} \leqq\{\Delta([\alpha(k), \beta(h)],[\alpha(k), \beta(k)])\}^{4} / 2^{8}$ whenever $1 \leqq h \leqq k-1$.

V. $\rho_{h} \in \bigcup_{i=1}^{k}[\alpha(i), \beta(i)]$ whenever $1 \leqq h \leqq n(k)$.

We construct the sequences as follows. Let $n(1)=1$ and choose $\varepsilon_{1}$ so as to satisfy I and II. Then III, IV, and V are trivially satisfied at this point. Now suppose the first $p$ terms of the sequences have been constructed so that $I$ through $\mathrm{V}$ are satisfied for $1 \leqq k \leqq p, 1 \leqq h \leqq p$. Then we construct the terms for $p+1$ by taking $n(p+1)$ to be the least integer for which

$$
\rho_{n(p+1)} \notin \bigcup_{i=1}^{p}[\alpha(i), \beta(i)],
$$

and choosing $\varepsilon_{p+1}>0$ small enough so that

$$
\begin{aligned}
\varepsilon_{p+1} & \leqq \frac{1}{2^{p+1+1}} \\
-\pi & <\rho_{n(p+1)}-\frac{\pi}{2} \varepsilon_{p+1}^{2}<\rho_{n(p+1)}+\frac{\pi}{2} \varepsilon_{p+1}^{2}<\pi \\
\frac{\pi}{2} \varepsilon_{p+1}^{2}+4 \varepsilon_{p+1}^{1 / 4} & \leqq \frac{1}{2} \min _{k \leqq p} \Delta\left(\rho_{n(p+1)},[\alpha(k), \beta(k)]\right) .
\end{aligned}
$$


It is easy to check that I through $\mathrm{V}$ are then satisfied for $1 \leqq k \leqq p+1,1 \leqq h \leqq p+1$; so the sequences can be constructed in accordance with I through $\mathrm{V}$.

Let $\chi_{n}$ denote the characteristic function of expi $[\alpha(n), \beta(n)]$ and let $\chi$ be the characteristic function of

$$
\bigcup_{n=1}^{\infty} \operatorname{expi}[\alpha(n), \beta(n)]
$$

Then $\chi=\sum_{n=1}^{\infty} \chi_{n}$. Set

$$
f_{n}\left(r e^{i \theta}\right)=\frac{1}{2 \pi} \int_{-\pi}^{\pi} \chi_{n}\left(e^{i t}\right) P_{r}(\theta-t) d t
$$

and set

$$
f\left(r e^{i \theta}\right)=\frac{1}{2 \pi} \int_{-\pi}^{\pi} \chi\left(e^{i t}\right) P_{r}(\theta-t) d t .
$$

Then $f$ is a bounded harmonic function in $D$. By the Lebesgue monotone convergence theorem, $f=\sum_{n=1}^{\infty} f_{n}$ (pointwise).

Let

$$
\Omega_{n}=\left\{r e^{i \theta}: 1-\varepsilon_{n}<r<1 \text { and } \Delta(\theta,[\alpha(n), \beta(n)])<(1-r)^{1 / 4}\right\} .
$$

It is easily seen that $\Omega_{n}$ is a Jordan domain in $D$ and that $\bar{\Omega}_{n} \cap C=\operatorname{expi}[\alpha(n), \beta(n)]$. By using IV one can easily show that $\bar{\Omega}_{n} \cap \bar{\Omega}_{m}$ is empty when $n \neq m$. I assert that

$$
0 \leqq f_{n}(z) \leqq \varepsilon_{n} \quad \text { whenever } z \in D-\Omega_{n} .
$$

That $f_{n}(z) \geqq 0$ follows from the fact that $P_{r}(\theta)$ is nonnegative. To prove the other inequality, take any $r e^{i \theta} \in D-\Omega_{n}$. Then either $r \leqq 1-\varepsilon_{n}$, or else $r>1-\varepsilon_{n}$ and $\Delta(\theta,[\alpha(n), \beta(n)]) \geqq(1-r)^{1 / 4}$. First suppose $r \leqq 1-\varepsilon_{n}$. Then

$$
\begin{aligned}
f_{n}\left(r e^{i \theta}\right) & =\frac{1}{2 \pi} \int_{\alpha(n)}^{\beta(n)} \frac{1-r^{2}}{(1-r)^{2}+2 r(1-\cos (\theta-t))} d t \\
& \leqq \frac{1}{2 \pi} \int_{\alpha(n)}^{\beta(n)} \frac{1-r^{2}}{(1-r)^{2}} d t=\frac{\beta(n)-\alpha(n)}{2 \pi} \frac{1+r}{1-r} \\
& =\frac{1}{2} \varepsilon_{n}^{2} \frac{1+r}{1-r} \leqq \frac{1}{2} \varepsilon_{n}^{2} \frac{2}{\varepsilon_{n}}=\varepsilon_{n} .
\end{aligned}
$$

On the other hand, suppose that $r>1-\varepsilon_{n}$ and $\Delta(\theta,[\alpha(n), \beta(n)]) \geqq(1-r)^{1 / 4}$. Then, for each $t \in[\alpha(n), \beta(n)]$ and each integer $m$, we have $|\theta-t+2 m \pi| \geqq(1-r)^{1 / 4}$, and hence, since $r>1-\frac{1}{4}$,

$$
\begin{aligned}
\cos (\theta-t) \leqq \cos (1-r)^{1 / 4} & \leqq 1-\frac{\left[(1-r)^{1 / 4}\right]^{2}}{2 !}+\frac{\left[(1-r)^{1 / 4}\right]^{4}}{4 !} \\
& \leqq 1-\frac{1}{4} \sqrt{ }(1-r) \quad(t \in[\alpha(n), \beta(n)]) .
\end{aligned}
$$


Therefore

Thus

$$
\begin{aligned}
f_{n}\left(r e^{i \theta}\right) & =\frac{1}{2 \pi} \int_{\alpha(n)}^{\beta(n)} \frac{1-r^{2}}{(1-r)^{2}+2 r(1-\cos (\theta-t))} d t \\
& \leqq \frac{1}{2 \pi} \int_{\alpha(n)}^{\beta(n)} \frac{1-r^{2}}{(1-r)^{2}+\frac{1}{2} r \sqrt{ }(1-r)} d t \\
& =\frac{1}{2} \varepsilon_{n}^{2} \frac{(1+r)(1-r)}{(1-r)^{2}+\frac{1}{2} r \sqrt{ }(1-r)} \leqq \frac{1}{2} \varepsilon_{n}^{2} \frac{2(1-r)}{\frac{1}{2} r \sqrt{ }(1-r)} \\
& \leqq \varepsilon_{n}^{2} \frac{1-r}{\frac{1}{2} \sqrt{ }(1-r)} \leqq \varepsilon_{n} \sqrt{ }(1-r) .
\end{aligned}
$$

(2) $f_{n}\left(r e^{i \theta}\right) \leqq \varepsilon_{n} \sqrt{ }(1-r)$ when $r>1-\varepsilon_{n}$ and $\Delta(\theta,[\alpha(n), \beta(n)]) \geqq(1-r)^{1 / 4}$. Inequality (2) completes the proof of (1), and, in addition, it shows that

(3) $f_{n}(z)$ approaches 0 as $z=r e^{i \theta}$ approaches any point of $C$ along any arc that does not meet $\Omega_{n}$.

Now, $\chi$ is continuous on expi $(\alpha(n), \beta(n))$, so (by (ii)) $f$ has general limit 1 at each point of expi $(\alpha(n), \beta(n))$. From this fact it is easy to deduce that there exists an arc at each of the points $e^{i \alpha(n)}, e^{i \beta(n)}$ along which $f$ approaches 1 . Thus $f$ has the asymptotic value 1 at each point of expi $[\alpha(n), \beta(n)]$.

Take any $\zeta \in C-\bigcup_{n=1}^{\infty}$ expi $(\alpha(n), \beta(n))$. By the lemma, there exists an arc $\gamma$ at $\zeta$ that does not meet any of the domains $\Omega_{n}$. By (1), and the fact that $\varepsilon_{n} \leqq 1 / 2^{n+1}$,

$$
\begin{aligned}
|f(z)| & \leqq \sum_{n=1}^{m}\left|f_{n}(z)\right|+\sum_{n=m+1}^{\infty}\left|f_{n}(z)\right| \\
& \leqq \sum_{n=1}^{m}\left|f_{n}(z)\right|+\frac{1}{2^{m+1}} \quad \text { whenever } z \in \gamma .
\end{aligned}
$$

By (3), each $f_{n}(z)$ approaches 0 along $\gamma$, so (4) shows that

$$
\limsup _{z \rightarrow \zeta ; z \in r}|f(z)| \leqq \frac{i}{2^{m+1}} \text {. }
$$

Since $m$ can be arbitrary, $f(z)$ approachés 0 along $\gamma$. Thus $f$ has the asymptotic value 0 at each point of

$$
C-\bigcup_{n=1}^{\infty} \operatorname{expi}(\alpha(n), \beta(n))
$$

We note that $f$ has both the asymptotic values 0 and 1 at each of the points $e^{i \alpha(n)}$, $e^{i \beta(n)}$, so that these points are ambiguous. If we set

$$
\begin{array}{ll}
\phi(\zeta)=1, & \zeta \in \bigcup_{n=1}^{\infty} \operatorname{expi}(\alpha(n), \beta(n)), \\
\phi(\zeta)=0, & \zeta \in C-\bigcup_{n=1}^{\infty} \operatorname{expi}[\alpha(n), \beta(n)], \\
\phi(\zeta)=1, & \zeta=e^{i \beta(n)} \quad \text { for some } n, \\
\phi(\zeta)=0, & \zeta=e^{i \alpha(n)} \quad \text { for some } n,
\end{array}
$$


then $\phi$ is a boundary function for $f$, so it only remains to prove that $\phi$ is not of Baire class 0 or 1 . Let

$$
A=\left\{e^{i \alpha(n)}: n=1,2,3, \ldots\right\}, \quad B=\left\{e^{i \beta(n)}: n=1,2,3, \ldots\right\} .
$$

It follows from $\mathrm{V}$ that each $\rho_{n}$ is in $\bigcup_{n=1}^{\infty}[\alpha(n), \beta(n)]$, so $\bigcup_{n=1}^{\infty} \operatorname{expi}[\alpha(n), \beta(n)]$ is dense in $C$. From this fact, and the fact that the closed arcs expi $[\alpha(n), \beta(n)]$ are pairwise disjoint, it is easy to deduce that $A$ and $B$ are each dense in $A \cup B$, and that $A \cup B$ has no isolated points. Thus $\mathrm{Cl}(A \cup B)$ is a perfect set. But $\phi$ takes the value 0 at each point of $A$ and the value 1 at each point of $B$, so the restriction of $\phi$ to $\mathrm{Cl}(A \cup B)$ can have no point of continuity. By the famous theorem of R. Baire $[2$, p. 88] $\phi$ cannot be of Baire class 0 or 1 .

THEOREM. Let $f$ be a harmonic function in $D$ such that, for some $p>1$,

$$
\left\{\int_{-\pi}^{\pi}\left|f\left(r e^{i \theta}\right)\right|^{p} d \theta: r \in[0,1)\right\}
$$

is a bounded set of numbers. Let $A$ be the set of points on $C$ at which fhas an asymptotic value, and let $G$ be the set of points on $C$ at which the general limit of $f$ exists. Then $A-G$ is a set of the first category.

Proof. Let $S$ be the set of points on $C$ at which the radial limit of $f$ exists. Then $S \subseteq A, C-S$ has measure 0 , and, if we define $\psi(\zeta)$ to be the radial limit of $f$ at $\zeta$ for each $\zeta \in S$, then $f$ is the Poisson integral of $\psi$. We can extend $\psi$ to a boundary function $\psi_{1}$ of $f$ that is defined on $A$. By the theorem on boundary functions for continuous functions, there exists a function $\phi$ on $A$ that is of Baire class 0 or 1 and differs from $\psi_{1}$ on at most a countable set. But then, since $\phi(\zeta)=\psi(\zeta)$ for almost every $\zeta, f$ is the Poisson integral of $\phi$. By the version of Baire's theorem on pointwise limits of continuous functions that is stated in Kuratowski's book [5, p. 301] (this reference was provided by the referee), there exists a set $K \subseteq A$ such that $A-K$ is of the first category and $\phi$ is continuous at each point of $K$. By (ii) and (iii), $K \subseteq G$, so $A-G$ is of the first category.

COROLlaRY. Let $f$ be a harmonic function in $D$ such that, for some $p>1$,

$$
\left\{\int_{-\pi}^{\pi}\left|f\left(r e^{i \theta}\right)\right|^{p} d \theta: r \in[0,1)\right\}
$$

is a bounded set. Let $\phi$ be a boundary function for $f$ defined on a set $E \subseteq C$. Then the set of discontinuity points (in $E$ ) of $\phi$ is of the first category relative to $C$.

Proof. Obviously $\phi$ is continuous at each point of $E$ where the general limit of $f$ exists.

The same results hold for analytic functions, as can be seen by applying the above theorem and corollary to the real and imaginary parts. The example of Bagemihl 
and Piranian [1, Theorem 6] shows that in the above theorem and corollary the hypothesis on the integrals

$$
\int_{-\pi}^{\pi}\left|f\left(r e^{i \theta}\right)\right|^{p} d \theta
$$

cannot be omitted.

\section{REFERENCES}

1. F. Bagemihl and G. Piranian, Boundary functions for functions defined in a disk, Michigan Math. J. 8 (1961), 201-207.

2. R. Baire, Leçons sur les fonctions discontinues, Gauthier-Villars, Paris, 1905.

3. K. Hoffman, Banach spaces of analytic functions, Prentice-Hall, Englewood Cliffs, N. J., 1962.

4. T. J. Kaczynski, Boundary functions for functions defined in a disk, J. Math. Mech. 14 (1965), 589-612.

5. C. Kuratowski, Topologie, Vol. I, 3rd ed., Monogr. Mat., Warsaw, 1952.

6. J. E. McMillan, Boundary properties of functions continuous in a disc, Michigan Math. J. 13 (1966), 299-312.

7. M. H. A. Newman, Elements of the topology of plane sets of points, second ed., Cambridge Univ. Press, New York, 1964.

8. L. E. Snyder, Bi-arc boundary functions, Proc. Amer. Math. Soc. 18 (1967), 808-811.

$$
\begin{array}{r}
\text { University of California, } \\
\text { Berkeley, California }
\end{array}
$$

